\title{
Anotaciones sobre el crecimiento en cautiverio de crías y juveniles de cocodrilo de río (Crocodylus acutus)
}

\section{Carta al Editor}

Fabio G. Cupul-Magaña, Pablo S. Hernández-Hurtado, Bartolo Cruz-Romero, Armando Rubio-Delgado.

Departamento de Ciencias. Centro Universitario de la Costa, Universidad de Guadalajara, Puerto Vallarta, Jalisco, México.

Se presentan los resultados de crecimiento en cautiverio de crías y juveniles de cocodrilo de río (Crocodylus acutus), dentro de las instalaciones de la Unidad para la Conservación, Manejo y Aprovechamiento Sustentable de la Vida Silvestre (UMA) "Reptilario Cipactli" del Centro Universitario de la Costa, clave INE/CITES/ DGVS-CR-IN-JAL/00, en Puerto Vallarta, Jalisco.

En total, se registró el crecimiento en el tiempo de 77 ejemplares repartidos en cuatro grupos de crías y un grupo de juveniles. Las crías se asignaron a tres acuaterrarios. En el acuaterrario No. 1, con una superficie de $9.32 \mathrm{~m}^{2}$, ingresaron 17 crías el 3 de febrero del 2001, muriendo dos en el transcurso del estudio. En el acuaterrario "A", con una superficie de $7.5 \mathrm{~m}^{2}$, ingresaron 22 crías el 20 de julio del 2001 y 8 crías el 10 de agosto del mismo año; se tuvo el deceso de tres organismos del primer grupo. En el acuaterrario "B", de igual superficie que el "A", ingresaron 26 crías el 20 de julio del 2001; muriendo cuatro ejemplares a lo largo del trabajo. Las crías asignadas a estos acuaterrarios se colectaron en la laguna Boca Negra, localizada en la porción norte de la mancha urbana de Puerto Vallarta, como parte del programa de protección de crías de cocodrilo de río en la Bahía de Banderas, avalado por el Instituto Nacional de Ecología. Las crías de los acuaterrarios "A" y "B", fueron de las camadas nacidas en libertad en el mes de julio del 2001 y las del acuaterrario No.1, fueron de las camadas nacidas en julio del 2000.

Finalmente, en el acuaterrario No. 2, se ubicaron a una serie de 13 cocodrilos juveniles (con edades entre dos y cinco años) que ingresaron en distintos tiempos a la UMA y que provenían de diversas localidades de la bahía y de la costa de Jalisco y Nayarit. Dentro de este acuaterrario de $15.25 \mathrm{~m}^{2}$ de superficie, ingresó un ejemplar el $4 \mathrm{de}$ noviembre de 1999 y, los doce restantes, los días 11 de marzo, 11 de mayo, 7 y 20 de junio, 10 de julio, 21 de agosto, 26 de septiembre, así como el 4 y 10 de octubre del 2000. Dos de estos

Solicitud de sobretiros: M. en C. Fabio G. Cupul-Magaña, Departamento de Ciencias. Centro Universitario de la Costa, Universidad de Guadalajara. Av. Universidad de Guadalajara No. 203, Delegación Ixtapa, C.P. 48280, Puerto Vallarta, Jalisco, México. 


\section{A Cervera-Andrade.}

cocodrilos, fueron liberados en su ambiente natural los días 7 de junio y 1 de agosto del 2001.

A los cocodrilos se les alimentaba al mediodía, con pescado marino molido o en trozos dos veces por semana; en raciones que comprendían el $33 \%$ de su peso corporal para los ejemplares de los acuaterrarios "A" y "B"; el 27.69\% para los del acuaterrario No. 1; y, del 6.27 al $12.29 \%$ para los del acuaterrario No.2. Es importante hacer notar que a las crías del acuaterrario "B" se les proporcionó como prueba un suplemento alimenticio modificado de una dieta cubana, que incluyó, dentro de un hemolizado (sangre de res), calcio (Magnafoscal), Febendazol como antiparasitante, Cloramfenicol como antibiótico, Dextrosa al 5\% como energético y polivitaminas (1).

Los registros biométricos se realizaron cada dos meses; estos incluían la medición de la longitud total de cuerpo ( $\mathrm{Lt}$; medida en centímetros desde la punta del hocico hasta la punta de la cola), el largo del hocico (Lh; medido en centímetros desde la base de los ojos hasta la punta del hocico) y el peso en gramos. El crecimiento de las crías de los acuaterrarios No. 1, "A" y "B", fue registrado hasta el 10 de noviembre del 2001, fecha en que fueron liberados en su ambiente natural; el correspondiente a los juveniles del acuaterrario No. 2, se tomó hasta el 13 de octubre del 2001 (estos cocodrilos aún continúan cautivos), salvo los dos que fueron liberados con anterioridad. En los acuaterrarios, la temperatura promedio ambiental y del agua a lo largo del trabajo fue de $29^{\circ} \mathrm{C}$.

$\mathrm{La} \mathrm{Lt}$ promedio de ingreso de los cocodrilos a los acuaterrarios fue de $54.68 \pm 2.02 \mathrm{~cm}$ para el No. 1; de $32.66 \pm 1.13 \mathrm{~cm}$ para el grupo del $20 \mathrm{de}$ julio y de $36.80 \pm 1.05$ para el grupo del 10 de agosto del "A"; de $31.16 \pm 2.00 \mathrm{~cm}$ para el "B"; así como entre 57.70 y $110.00 \mathrm{~cm}$ para los del No. 2. En el mismo orden, el peso promedio de ingreso fue de $402 \pm 58.7 \mathrm{~g}$ en el No. 1 ; de $87 \pm 5.3 \mathrm{~g}$ en el grupo del 20 de julio y de $125 \pm 14.0$ g en el grupo del 10 de agosto de "A"; de $81 \pm 9.0$ g en el "B"; así como entre 650 y 5,400 g en el No. 2 .
Como primer resultado, se logró obtener la relación entre las variables biométricas de $\mathrm{Lh}$ y Lt para las crías y juveniles de cocodrilo de río, a partir del siguiente modelo de regresión lineal simple: $\mathrm{Lt}$ $=9.52(\mathrm{Lh})+10.59$. Este modelo puede permitir conocer la longitud total del cocodrilo con sólo determinar la longitud total del hocico, siendo esta relación significativamente alta $(\alpha=5 \%)$, ya que se contó con un coeficiente de determinación de $\mathrm{r}^{2}=$ 0.98 (2).

De acuerdo con los incrementos registrados en talla y peso para los 77 organismos, se obtuvo que la tasa de crecimiento promedio diario en longitud total del cuerpo fue de $0.057 \pm 0.011 \mathrm{~cm} /$ día, es decir, $1.71 \mathrm{~cm} / \mathrm{mes}$; en cuanto a peso, ésta fue de $2.51 \pm 1.99 \mathrm{~g} /$ día o $75.30 \mathrm{~g} / \mathrm{mes}$. Los registros de talla se encuentran muy por debajo de los reportados para la especie en Chiapas de hasta $4.0 \mathrm{~cm}$ por mes (3); en Florida de $3.36 \mathrm{~cm}$ por mes, en los primeros 17 meses de vida (4) y, en general, para el grupo de los cocodrilos, cuyo promedio mensual es de $4.6 \mathrm{~cm}$ en los dos primeros años de vida (5). Seguramente, esto pueda ser un reflejo de las condiciones no adecuadas de cautividad en la UMA, resultado de variaciones bruscas en la temperatura del agua y el ambiente, la densidad de organismos por superficie o el diseño de los acuaterrarios, la técnica de manejo del alimento o, tal vez, resultado del metabolismo típico de esta población local, sólo comprobable al realizar mediciones de captura y recaptura en libertad a lo largo del tiempo.

Por último, no fue posible establecer diferencias significativas (Prueba t para la media; $\alpha=5 \%$ ) (2) con respecto al incremento en talla y peso experimentado por el grupo de crías con suministro de suplemento alimenticio (acuaterrario "B") y de aquellas a las que sólo se les alimentó con pescado (grupo del 20 de julio del acuaterrario "A").

Palabras clave: Crocodylus acutus, crecimiento, cautiverio, Puerto Vallarta.

\section{Revista Biomédica}




\section{REFERENCIAS.}

1.- Benavides-Calvo RR, Fonseca-Sanz RN. Enriquecimiento dietético y su efecto sobre el crecimiento del Crocodylus acutus en el zoocriadero de Manzanillo. Cuba: Empresa Nacional para la Conservación de la Flora y la Fauna; 1999. p. 11.

2.- Marques de Cantú MJ. Probabilidad y estadística para ciencias químico-biológicas. México: McGraw-Hill; 1991. p. 655 .

3.- Álvarez del Toro M. Los Crocodylia de México. México: IMERNAR; 1974. p. 70.

4.- Throbjarnarson JB. Ecology of the american crocodile, Crocodylus acutus. En: Crocodiles their ecology, management and conservation. IUCN Publication New Series. p. 228-259.

5.- Bolton M. La explotación del cocodrilo en cautividad. Italia: Guía FAO Conservación 22; 1994. p. 156. 